\section{Can ophthalmic requests for neuroimaging be improved?}

\begin{abstract}
Aim To review and set guidelines for all neuroradiological referrals from the ophthalmic service in a typical UK district general hospital.

Method Patients referred for diagnostic neuroimaging were identified from the radiology database over a 1-year period. A case note review was undertaken. The clinical indication for and results of neuroimaging performed were ascertained. The justification for neuroimaging was determined.

Results Of the total 31411 patients that were seen in the eye department, $103(0.32 \%)$ had diagnostic neuroradiological imaging performed. The indications for imaging were: suspected compressive lesion of the anterior visual pathway in 57 patients $(55.3 \%)$, acquired ocular motility disturbance in 20 patients (19.4\%), suspected orbital pathology in 11 patients $(\mathbf{1 0 . 6 \% )}$, cerebrovascular accident in nine patients $(8.7 \%)$, and six patients $(5.8 \%)$ were imaged for either headache or sinusitis. Radiological lesion detection rate was highest for cerebrovascular accident $\mathbf{( 8 8 . 8 \% )}$ and lowest acquired for ocular motility disturbance $(4.9 \%)$.

Conclusion Ophthalmic requests for diagnostic neuroimaging were found to be unnecessary in only $9 \%$ of patients. Guidelines for the referral of ophthalmic patients for neuroimaging are discussed. Eye (2004) 18, 290-292. doi:10.1038/ sj.eye. 6700627
\end{abstract}

Keywords: CT; MRI; ophthalmology; guidelines; audit; neuroimaging

SP Kelly

Bolton Hospitals NHS Trust Bolton, BL4 OJR, UK E-mail: simon.kelly@ boltonh-tr.nwest.hs.uk

Received: 10 September 2002

Accepted in revised form: 21 February 2003
Ophthalmology Bolton Hospitals NHS Trust

${ }^{2}$ Department of Radiology Bolton Hospitals NHS Trust Bolton, UK

Correspondence:
JP Mathews' ${ }^{1}$, D Mathews ${ }^{1}$, S Walker ${ }^{1}$, J Tuck² and SP Kelly' between various soft and bony tissues of the skull and orbit can be assessed. ${ }^{1,2,3}$ The main disadvantages of CT include poorer contrast resolution between different soft tissues relative to magnetic reasonance imaging (MRI), beam-hardening artefacts, lack of ability to scan directly in the sagittal plane, and high radiation exposure. Multislice spiral CT scanners now overcome some of the disadvantages of the older single slice scanners. This is of particular relevance in high-resolution multiplanar reconstructions. The radiation dose from a CT brain scan is approximately $2.3 \mathrm{mSv}$, which is equivalent to 115 chest $X$-rays or 1 year of natural background radiation. ${ }^{4}$ A recent European Union directive aims to reduce patients' overall radiological exposure by a considered approach towards only requesting appropriate interventions by clinicians. $^{5}$

MRI is more useful in localizing soft tissue abnormalities, vascular and inflammatory lesions. The advantages of MRI include lack of ionizing radiation, direct multiplanar imaging, increased sensitivity to pathology, increased tissue contrast, and no known adverse effects. Imaging time may sometimes exceed 15-20 min per study dependent on the number of sequences used. This makes it difficult to scan children and patients with movement disorders or claustrophobia unless sedation or general anaesthesia is used.

Cardiac pacemakers and implanted or retained metallic foreign bodies are an absolute contraindication for an MRI scan. Greater access to MRI in the UK is needed and should be the imaging modality of choice in patients with neuro-ophthalmologic disorders of suspected brainstem origin. ${ }^{6}$

The relative demand for and the exact role of neuroimaging in various different ophthalmic situations has not been clearly established. Guidelines for neuroimaging requests in ophthalmology are lacking. We therefore sought to audit the reasons for and appropriateness of

\section{Introduction}

Computed tomography (CT) has become a readily available, fast, and reliable imaging tool for the ophthalmologist. The anatomic relation 
diagnostic neuroradiology examinations in a typical UK District General Hospital.

\section{Method}

We undertook a retrospective study of ophthalmic patients undergoing diagnostic neuroimaging at Bolton Hospital NHS Trust. This hospital is located North-west of Manchester in England, serving a catchment area with approximately 250000 residents. Patients referred from the ophthalmic department for diagnostic neuroimaging from 1st December 1998 to 30th November 1999 were identified from the radiology database. In line with our local practice, all requests were made with consultant ophthalmologist's approval.

A case note review of all of these patients' records was undertaken during April and May 2000. A minimum of 5 months post neuroimaging clinical follow-up was required to be available for all patients. The clinical indications for and results of the neuroimaging performed were extracted. An assessment as to the justification and appropriateness for the diagnostic neuroimaging requested was determined at the time of case note review. A subjective consensus judgment was based on the patient's initial presentation and clinical evolution over the succeeding 5-18 months of clinical follow-up from the date of neuroimaging.

\section{Results}

During the 12-month study period, the total number of patients attending the eye unit was 31411 (comprising of 8455 new and 22956 follow-up). Of these, 103 (0.32\%) underwent neuroimaging and all these case notes were available for our present review. CT was performed in each case, with two patients undergoing subsequent MRI at a regional radiology centre. The radiology department performed a total of 3025 cranial neuroimaging studies during this time, of which $3.4 \%$ were the ophthalmic referrals.

Table 1 shows the various suspected clinical diagnoses of 103 ophthalmology patients undergoing neuroradiological investigations. The outcomes are grouped as being either clinically necessary or unnecessary. The number of patients with lesions detected are shown. In all, $22(21.3 \%)$ patients had reported abnormalities. The neuroradiological lesion detection rate was highest in patients with suspected cerebrovascular accident $(88.8 \%)$ and lowest in ocular motility disturbance (4.9\%). The clinical assessment prior to the request for neuroimaging with the knowledge of the subsequent clinical progress was judged satisfactory and the scan considered clinically necessary in 91 patients $(91.3 \%)$. Further clinical information in patients with paranasal sinus disease (three patients), retrobulbar neuritis (three patients), and visual field examination in patients with headache (three patients) in the first instance may have reduced the need for unnecessary neuroimaging in these nine patients $(8.7 \%)$.

\section{Discussion}

The introduction of CT scanning in the 1970s and MRI in the 1980s has revolutionized medical practice. In our department, the vast preponderance of CT over MRI scanning reflects the local waiting times of 1 month for CT and 12-18 months for MRI at the time of the study. This poor accessibility to MRI investigation is typical of many UK district general hospitals. Neuroimaging was requested for one in every 305 of ophthalmic patients attending within the study period. In our clinical setting, all scans were requested for diagnostic purposes. The suspicion of compressive lesion of the anterior visual pathway in 57 patients $(55.3 \%)$ was the commonest clinical reason for a diagnostic neuroradiological request, but also the most likely to give a normal scan result. In the three patients with a first attack of classical retrobulbar neuritis, CT scan performed at 4 weeks did not detect any abnormality. Visual recovery occurred 2-4 weeks after the scan. A longer period of ophthalmic observation in these patients may have obviated the need for CT scanning. MRI in this clinical situation may offer prognostic information regarding the development of possible future demyelination episodes.

In the group with acute ocular motility disturbance, CT failed to detect any identifiable lesions. MRI would have

Table 1 Outcomes of CT scans in 103 ophthalmic patients grouped by suspected clinical diagnosis

\begin{tabular}{|c|c|c|c|c|c|c|c|}
\hline Justification & $\begin{array}{c}\text { Lesion } \\
\text { detection }\end{array}$ & $\begin{array}{l}\text { Suspected lesion, } \\
\text { anterior visual } \\
\text { pathway }\end{array}$ & $\begin{array}{c}\text { Acquired ocular } \\
\text { motility } \\
\text { disturbance }\end{array}$ & $\begin{array}{l}\text { Orbital } \\
\text { lesion }\end{array}$ & $\begin{array}{c}\text { Cerebrovascular } \\
\text { accident }\end{array}$ & Headache & Sinusitis \\
\hline \multirow[t]{2}{*}{ Necessary } & Yes & 4 & 1 & 9 & 8 & 0 & 0 \\
\hline & No & 50 & 19 & 2 & 1 & 0 & 0 \\
\hline \multirow[t]{2}{*}{ Unnecessary } & Yes & 0 & 0 & 0 & 0 & 0 & 3 \\
\hline & No & 3 & 0 & 0 & 0 & 3 & 0 \\
\hline
\end{tabular}


been more appropriate for those cases that did not subsequently resolve spontaneously. However, this was not readily available locally at the time. In a Japanese study using diagnostic imaging, the abnormality detection rate was found to be high for supranuclear and myogenic palsy and the mechanical disturbance groups, but very low for the ocular motor nerve palsy groups. In supranuclear palsy patients, small infarctions of the brainstem were discovered by MRI but were not found with CT. MRI thus proved useful in detecting abnormalities of brainstem lesions. ${ }^{7}$

In our study, only $8.7 \%$ of scans requested for diagnostic purposes were judged inappropriate. We are aware of a similar audit by Sleep et al. ${ }^{8}$ They showed that, on setting local guidelines, inappropriate neuroimaging referrals were reduced from 12 to $2 \%$ in their department. Neuroimaging is one of the more expensive and potentially hazardous investigations requested by ophthalmologists. The potential hazards of radiation and the likelihood of obtaining useful results should be carefully considered before proceeding.

\section{Conclusions}

Neuroimaging requirement in a general ophthalmic department was 32 per 10000 ophthalmology patients attending in 1999. Most scan requests were appropriate. The clinical assessment might be improved upon and neuroimaging avoided in $8.7 \%$ of patients by following these suggested guidelines.

1. Suspected compressive lesions of the anterior visual pathway: Request neuroimaging if there is reduced vision, a reproducible visual field defect, pupillary defect, or suspicious optic disc. Trainee ophthalmologists should discuss cases with their supervisor.

2. Oculomotility disorder: Consider referral to a neurologist or neuro-ophthalmologist. CT with contrast or MRI may be more appropriate than plain CT. Cerebral angiography may be required.
3. Retrobulbar neuritis: Await improvement in typical cases. Request a scan, however, if there is no clinical improvement within 6-8 weeks or an atypical clinical picture. MRI of the brain and orbits is the investigation of choice both for localization of pathology and prognosis. There should, however, be discussion with a neurologist and informed discussion with the patient as to the potential implications of the diagnosis of multiple sclerosis.

4. Sinusitis: Do not request a scan in uncomplicated cases. Consider early ENT referral.

5. Headache: Do not request a scan in patients with simple isolated headache. Ensure visual field examination is performed. Consider a neurology referral.

\section{Acknowledgement}

This work was presented at the North of England Ophthalmic Society, Manchester, June 2000.

\section{References}

1 Gans M. Neuroimaging techniques for the ophthalmologist. Can J Ophthalmol 1997; 32(4): 277-281.

2 Slamovits TL, Gardner TA. Neuroimaging in neuro-ophthalmology. Ophthalmology 1989; 96(4): 555-568.

3 Grossman RI, Lynch RM. Neuroimaging in neuro-ophthalmology. Neurol clin 1983; 1(4): 831-857.

4 Royal college of Radiologists London. Guidelines for Doctors Making Use of Clinical Radiology, 4th Ed. 1998, p. 13.

5 Ionising Radiation (Medical Exposure) Regulations 2000, Statutory Instrument No. 1059, HMSO, 2000.

6 Lewis MA, Goldstein S, Baker RS. Magnetic resonance imaging of the posterior fossa in ocular motility disorders. Four case studies. J Clini Neuro Ophthalmol 1987; 7(4): 235-2409.

7 Kobashi R, Ohtsuki H, Hasebe S. Clinical studies of ocular motility disturbances. Part 1 . Ocular motility disturbances: causes and incidence. Jpn J Ophthalmol 1996; 40(4): 502-510.

8 Sleep T, Wirix M, Cole M, Debney N. The use of CT and MRI scanning within ophthalmology. In: Audit News (Winter Ed). London: The Royal College of Ophthalmologists, 2001; 9-10. 